\title{
A Research of Micromachining of Materials
}

\author{
Rakesh Kumar Singh, Shivaji Chaudhary
}

\begin{abstract}
The requirement for apparatus with surfaces and higher quality rates has been rising in businesses. That was an increasing need of operational services and products out of plastics, polymers, composites and alloys. The article provides an overview on micro-machining with accent in micromachining.
\end{abstract}

\section{INTRODUCTION}

Even the miniaturization of the apparatus is demanding the creation of elements in areas which have communications, electronic equipment, medicine, biotechnology, optics, along with even avionics, to list a couple. Particular applications contain microscale gas cells reactors necessitating pumps, detectors and apparatus microphones such as fiber production, micro-nozzles for jets, micromolds lithography masks, and also more [1].Like a response for the requirement, many micro- producing processes have lately emerged, including as for example for instance ray lithography electro-deposition molding (LIGA), deep reactive ion etching, deep UV lithography, electric discharge machining, laser machining and personal computer numerical controlled (CNC) micro-machining. The majority of those methods need expensive, high priced, or time consuming gear [2], thus one of those portable micro- fabricating approaches for developing 3 - dimensional (3-d) characteristics of alloys, polymers, ceramics, and composites is mechanical micro machining. Micro-machining mini turning, drilling and milling gears In diameter to create capabilities. Alnevertheless substance and geometric capacities of micro machining are attested by [3] the industrial applications of how all micro machining was hindered by the deficiency of expertise and comprehension regarding the micro-machinability of substances [4].

\section{FEATURES OF MICROMACHINING - MICROMILLING}

Micromilling, among of those mechanical micro machining procedures, can be just actually really a course of action which uses wind manufacturers which generally change in diameter from 100 to $500 \mathrm{~m}$ and also possess border radii that change from 1 to $10 \mathrm{~m}$. in addition, the micromilling procedure has a lot of conspicuous characteristics that distinguish it by your macro- end-milling procedure. Whilst the end milling procedure is scaled from normal dimensions (a hundred $\mathrm{m} /$ tooth feed prices, inch millimeter depths of cuts) into micro-endmilling dimensions (inch $\mathrm{m} /$ tooth feed prices, $100 \mathrm{~m}$ depths of trimming), unique

Revised Version Manuscript Received on 30 May, 2018.

Rakesh Kumar Singh, Mechanical Engineering, Noida Institute of Engineering and Technology, Noida, Uttar Pradesh, India.

(Email: research.paper@niet.co.in)

ShivajiChaudhary, Mechanical Engineering, Noida Institute of Engineering and Technology, Noida, Uttar Pradesh, India.

(Email: research.paper@niet.co.in) occurrences dominate the micro-endmilling course of action in comparison to people typically found in normal milling [5]. Said the difference among grinding arises thanks to the scale of their surgery. Nevertheless, feed to the radius of this cutter's proportion is in micromilling increased. In addition, this tool tip's run out, also inside of just microns influences the truth of both microfilming rather than this milling. A processor depth is depended upon by the processor creation from micromilling and the processor is not formed application and work piece are participated rather compared to milling. The instrument deflection from the micromilling influences precision and the processor development of this surface when compared with traditional milling. The application edges) $\mathrm{n} d \mathrm{I}$ s $\mathrm{n}$ I f o r m y combined the border are tremendously essential whilst the processor depth becomes a comparable into this border radius [7]. Due to the fact the processor load is modest when compared with this cutting border radius, the measurement effect also plowing compels be important on surface and induce production. Micromilling can lead to surface creation as a result of plowing- controlled cutting on and unwanted stream of this stained material as soon as the border gets exploited along with blunter. There are happenings in micromilling from being implemented to it 19 , that forbid the outcomes of milling. It can't be presumed the microstructure of this material is [5]. Its own effect grows crucial Since application size gets smaller. Inside this job it $\%$ effect wasn't supposed. Secondly, the very border radius' result isn't slight: the processor is affected by it. Minimum chip depth is now a part with This parameter and decides that the Transition involving 2 cutting edge requirements; exactly wherever processors are generated and at which plowing happens [6].

\section{PARAMETERS IN MICROMACHINING\& RESULTS}

The machining parameters in micromilling have been speed, feed speed and feed each evening. The literature indicates that studies are performed to reveal around that degree those parameters to help determine the impacts about the application along with the essence of the components. Back in Fig. inch per record of those inputs and affects from micromilling are proven.Back in 2008, Filiz et al. [8] researched the employment of this mechanical micromilling approach for the manufacture of micro-scale piercing ingredient in biocompatible substances. The writers applied the two habits produced, exclusive geometry, resources together with cutting edge diameters $254 \mathrm{~m}$ and $101.6 \mathrm{~m}$. To inquire into the repercussions of feed speed, rate, and also axial thickness of cut the operation of the gear, a style of experiments analysis has been con- based on 
polymethylmethacrylate (PMMA). They concluded the spindle rate gets getting the absolute most promi-impact for a great many induce elements, also by a growth has resulted in by growth in rate. \% experimental and modeling scientific tests on meso/micro- design of AISI 4340 metal and AL 2024-T6 aluminum to foresee temperature areas as well as chip development. Additionally they tested processor depth and size impacts[9]. To run this particular analysis, the writers utilized a 2-flute tungsten carbide about cobalt matrix WC-Copercent and 3.175 $\mathrm{MM}$ and also a predetermined spindle rate of $60000 \mathrm{rpm}$. Cutting rate utilized was 22.62 $\mathrm{m} / \mathrm{min}$ and also $59.85 \mathrm{~m} / \mathrm{min}$ and nourish varied in the 0.265 $\mathrm{m}$ to $4 \mathrm{~m}$. Substantial induce variations had been detected whilst the width of the cutter lessened and also the spindle rate rose. As a way to review the effect of this tool border requirement and also the work-piece microstructure," Vogler along with his coworkers [10] at 2004 conducted experiments using 508 there's diameter stop manufacturers on work-piece substances with various micro-structures within a reach of feed prices. Four substances had been selected to its experimentation; 2 particularly ready, single-phase substances (pure ferrite and pearlite) and 2 multi-phase substances with various compositions of their 2-phase substances [8]. They conducted 5 M M Extended End milling cuts beneath states as a way to examine the interaction among plowing and course of action status impacts. The single-axis single-axisquoted from the manufacturer $\mathrm{m}$. The micro wind manufacturers used through the experimentation ended up micro-grain tungsten carbide (WC) resources, made by diamond milling, two-fluted along also a $254 \mathrm{~m}$ diameter and also a 30helix angle. This study comprised full-immersion (slot) chopping using compacted thickness of reduction of thirty m. 4 feed speeds $(0.75,1.5,3,5$ also $6 \mathrm{~m} /$ flute $)$ and also three cutting rates (forty; eighty, along with also $120 \mathrm{~m} / \mathrm{min}$ ) had been believed inside this experiment. Feed rates' variety have been picked to incorporate exactly the plowing, plowingion, along with also chip depth impacts from the info. Just lately, Davim and Cardoso [12] Davisrder to do an all-inclusive study on the surface roughness of the surfaces parameters like machining plans in addition to feed speed are diverse into marketing micromilling. Al 2011 aluminum utilized. It's definitely really metal known because of its own traits and mechani- centric attributes. The software was used to machine the work piece proved to be a K-10, 0,8 millimeter diameter end mills. 4 feed speeds (two, 6, 4, also 8m/flute) plus yet another spindle rate $6,500 \mathrm{rpm}$ had been believed inside this experimentation. About three machines- centric approaches are utilized: continuous spiral andseriallelzig-zag. Even the burrs developed together using all the 2nd plan (level - called spiral) are substantially pronounced[11]. The stunt plan that is continual has been that the sole who presented with that the effect. Floor roughness profiles and also the worth of RzD (Me-an peak-to-valley Peak - DIN4768)

\section{CONCLUSIONS}

The absolute most important input signal from the micro-machining pre Jesus is regarded as the various tools (traits and substances), machine resources (spindle technological innovation, toolholder, rigidity) and not least significantly, machining parameters (cutting speed, feed speed and feed each tooth). About the flip side, the problems advert - equipped together with micro-machining would be the minimal processor depth and measurement impact, the chopping temperatures and cutting powers that have an effect on the application usage and also its own particular collapse, and this, subsequently, have an effect on the burr for- mation and, thus, area high excellent.

\section{REFERENCES}

1. D. Nocedo-Mena et al., "Modeling Antibacterial Activity with Machine Learning and Fusion of Chemical Structure Information with Microorganism Metabolic Networks," J. Chem. Inf. Model., vol. 59, no. 3, SI, pp. 1109-1120, Mar. 2019.

2. V. Beya-Marshall, J. Herrera, F. Santibanez, and T. Fichet, "Microclimate modification under the effect of stationary and portable wind machines," Agric. For. Meteorol., vol. 269, pp. 351-363, May 2019.

3. D. Agrawal and D. Kamble, "Effect and optimization of photochemical machining process parameters for manufacturing array of micro-hole,” J. BRAZILIAN Soc. Mech. Sci. Eng., vol. 41, no. 4, Apr. 2019.

4. H. Ogi, S. Moriwaki, M. Kokubo, Y. Hikida, and K. Itoh, "Label-free classification of neurons and glia in neural stem cell cultures using a hyperspectral imaging microscopy combined with machine learning," Sci. Rep., vol. 9, Jan. 2019.

5. Z. Xu, Y. Chang, Y. Chai, H. Wang, and R. Yuan, "Ultrasensitive Electrochemiluminescence Biosensor for Speedy Detection of microRNA Based on a DNA Rolling Machine and Target Recycling," Anal. Chem., vol. 91, no. 7, pp. 4883-4888, Apr. 2019.

6. Z.-L. Wang and Y. Adachi, "Property prediction and properties-to-microstructure inverse analysis of steels by a machine-learning approach," Mater. Sci. Eng. A-STRUCTURAL Mater. Prop. Microstruct. Process., vol. 744, pp. 661-670, Jan. 2019.

7. C.-F. J. Kuo, C.-P. Tung, and W.-H. Weng, "Applying the support vector machine with optimal parameter design into an automatic inspection system for classifying micro-defects on surfaces of light-emitting diode chips," J. Intell. Manuf., vol. 30, no. 2, pp. 727-741, Feb. 2019.

8. G. Mata et al., "Automated Neuron Detection in High-Content Fluorescence Microscopy Images Using Machine Learning," Neuroinformatics, vol. 17, no. 2, pp. 253-269, Apr. 2019.

9. D. Garbiec and P. Siwak, "Microstructural evolution and development of mechanical properties of spark plasma sintered WC-Co cemented carbides for machine parts and engineering tools," Arch. Civ. Mech. Eng., vol. 19, no. 1, pp. 215-223, Mar. 2019.

10. V. Dubey, A. Ahmad, A. Butola, D. Qaiser, A. Srivastava, and D. S. Mehta, "Low coherence quantitative phase microscopy with machine learning model and Raman spectroscopy for the study of breast cancer cells and their classification," Appl. Opt., vol. 58, no. 5, pp. A112-A119, Feb. 2019.

11. Y. Wang, B. Zou, and C. Huang, "Tool wear mechanisms and micro-channels quality in micro-machining of Ti-6Al-4V alloy using the Ti(C7N3)-based cermet micro-mills," Tribol. Int., vol. 134, pp. 60-76, Jun. 2019. 